\title{
Resistance of velocity slip flow in pipe/channel with a sudden contraction
}

\author{
Qiangqiang Sun (孙强强), ${ }^{1}$ Kwing-So Choi, ${ }^{1}$ Yong Zhao (赵勇), ${ }^{2}$ and Xuerui Mao (毛雪瑞) ${ }^{1}$, a) \\ ${ }^{1)}$ Faculty of Engineering, University of Nottingham, Nottingham, NG7 2RD, UK. \\ 2) AVIC Aerospace Systems, Chaoyang District, Beijing, 100028, China.
}

(Dated: 2 June 2020)

A novel approach based on the local entropy generation rate, also named as the second law analysis (SLA), is proposed to compute and visualize the flow resistance in mass transfer through pipe/channel with a sudden contraction component (SCC) at low Reynolds number $(R e)$ featuring velocity slip. The linear Navier velocity slip boundary condition is implemented using the explicit scheme. At small Reynolds number, i.e. $R e \leq 10.0$, the flow resistance coefficient of the SCC, $K_{S C C}$, is found to be a function of the dimensionless velocity slip length $L_{\text {slip }}^{*}$ and $R e^{-1}$, and gradually increase to a constant value at contraction ratio $R_{\text {area }} \geq 8$, reaching a formula $K_{S C C}=\left(0.4454 L_{\text {slip }}^{* 3}-1.894 L_{\text {slip }}^{* 2}+2.917 L_{\text {slip }}^{*}+\right.$ $8.909) / R e$. Over this range of $R e$, the equivalent length of the flow resistance is almost independent on $R e$, while out of this range, the equivalent length increases monotonically with $R e$. Moreover, the dimensionless drag force work around the SCC is negative and reaches a minimum at a critical $L_{\text {slip. }}^{*}$. The SLA reveals that the regions affected by the SCC mainly concentrate around the end section of the upstream pipe/channel rather than the initial partition of the downstream section reported in large $R e$ turbulent flow, and this non-dimensional affected upstream length increases with $L_{\text {slip }}^{*}$. The fluid physics are further examined using SLA to evaluate the energy loss over the entire domain, decomposed as the viscous dissipation inside the domain and the drag work on the wall boundary.

\section{INTRODUCTION}

The determination of the flow resistance in low Reynolds number $(R e)$ mass transfer through micro and nanoscale pipes and channels with sudden contraction components (SCC) is a vital issue in the process of design and fabrication of efficient microfluidic devices (i.e., micro-electro-mechanical systems ${ }^{1}$, heat exchangers ${ }^{2}$ and cooling of electronic chips ${ }^{3}$ ). For fully developed laminar pipe flow without velocity slip, it is known that the Darcy friction factor (flow resistance coefficient is proportional to the friction factor in a straight pipe/channel) is $64 / R e^{4-8}$. This value was also reported in the well-known Moody diagram, stressed by Moody as independent on the wall relative roughness in laminar flow ${ }^{9} . \mathrm{Kim}^{10}$ reported that this value can be predicted by the classic macroscopic theory in microscale flow and concluded that scale effects do not influence the flow resistance coefficient.

However, a number of experimental measurements of the flow resistance in the laminar flow regime do not agree with each other and even show significant discrepancies ${ }^{11}$. After performing a series of experiments, Qu et al. ${ }^{12}$ observed that the friction factor of laminar flow in microchannels with various shapes and dimensions is larger than the one obtained from the macroscale theory. On the contrary, the friction factor was found to be less than its macroscale counterpart by other researchers ${ }^{13-17}$. There have been no conclusive explanations of these significant discrepancies. Some works attributed these to the flow separation ${ }^{18}$, the dependence of viscosity on the channel size or other physical effects which become significant in small-scale ducts ${ }^{19}$ and so on. We believe that the following factors are also involved:

Firstly, the non-slip boundary condition on the wall becomes less valid as a significant velocity slip has been

\footnotetext{
a)Electronic mail: xuerui.mao@ nottingham.ac.uk
}

observed $^{20-23}$ when the characteristic length of the channel is reduced. This is because the fluid slips when the molecular attraction between the fluid and the solid surface is reduced as the free surface energy of the solid becomes very low. Watanabe et al. ${ }^{24}$ conducted experiments on water flow through square and rectangular ducts and pipes with highly water-repellent walls producing velocity slip boundary conditions and observed the maximum drag reduction ratio could reach up to $20 \%$ for square ducts and $14 \%$ for pipes. This observation offers potential explanations on the overestimation of the friction factor at extremely small scales by the macroscale theory aforementioned in the literature.

Also, the friction factor of laminar flow in micro-level ducts has been reported to be dependent on the relative roughness of the solid wall ${ }^{25}$, contradicting the Moody diagram ${ }^{9}$. Gloss et al. ${ }^{25}$ conducted experiments to test laminar flow in microchannels with length $130 \mu \mathrm{m}$, height from $20 \mu \mathrm{m}$ to $400 \mu \mathrm{m}$ and relative roughness ranging from 0 to 1 . Their outcomes demonstrate that the classic macroscale no-slip theory underestimates the friction factor because an increased dissipation rate near the roughness element causes more pressure loss compared with smooth wall surfaces.

Flow resistance is also dependent on the geometry variations, such as an SCC, as has been investigated in macroscale flow with applications including injection moulding, extrusion, thermoforming and polymer melt processing. A wide range of investigations including theoretical analyses ${ }^{26}$, numerical simulations ${ }^{27}$ and experiments ${ }^{28}$ were conducted for flow in an SCC with various contraction ratios. It should be pointed out that almost all these studies were on the effects of contraction ratio or wall slip on the flow behaviors (i.e., size and intensity of vortex, vortex shape and streamlines) of non-Newtonian fluids. No studies on the flow resistance coefficient of contraction pipe/channel flow of Newtonian fluids with a wall slip boundary condition have been performed to the best knowledge of the authors.

The flow resistance coefficient is commonly computed 
from pressure loss by solving the Bernoulli or Navier-Stokes (NS) equations. Celata et al. ${ }^{29}$ observed that the viscous heating could be applied to determine the resistance coefficient of microchannel no-slip flow and validated this in their experiments. Naterer et al. ${ }^{30}$ reported that the loss of available work could be directly related to the entropy generation by comparing the viscous dissipation term in the mechanical energy equation with the volumetric rate of entropy generation equation firstly proposed by Bejan ${ }^{31}$. The method based on the entropy generation is called the second law analysis (SLA), referring to the second law of thermodynamics. The SLA has been widely used in the optimal design of fluid engineering systems ${ }^{32}$, analyzing the flow resistance for no-slip flow, where the drag work on the surface is zero ${ }^{33}$. Compared with the common NS method, the SLA gives a direct physical interpretation of the flow resistance by revealing the distribution of the energy loss over the entire flow domain, as well as the drag work along the surface.

The main objective of the present study is to quantitatively examine the flow resistance coefficient and reveal the physics in flow through a microscale SCC with a linear Navier velocity slip boundary condition (SBC). In Section II, we illustrate the details of the mathematical formulation of the linear SBC and the analytical results of the resistance coefficient of flow in a plane parallel channel from the view of exact solutions of the NS and SLA approaches, followed by validations by numerical simulations. Corresponding simulation details, flow resistance coefficients and two factors causing flow resistance (the wall drag force work and the fluids viscous dissipation) for flow through an SCC are then described in Section III. Finally, conclusions are drawn in Section IV.

\section{RESISTANCE OF VELOCITY SLIP FLOW IN A 2D PLANE CHANNEL}

\section{A. The Navier velocity slip wall boundary condition}

The incompressible steady Newtonian flow through a plane parallel channel as shown in Fig.1a is governed by the NS equations $^{34}$ :

$$
\begin{aligned}
& \nabla \bullet \boldsymbol{U}=0, \\
& \nabla \bullet(\boldsymbol{U} \otimes \boldsymbol{U})=-\nabla \frac{p}{\rho}+\nabla \bullet\left(\frac{\mu}{\rho} \nabla \boldsymbol{U}\right) .
\end{aligned}
$$

where $\boldsymbol{U}$ is the velocity vector, $\boldsymbol{U} \otimes \boldsymbol{U}=\boldsymbol{U} \bullet \boldsymbol{U}^{T}, p$ is the pressure, $\rho$ is the density and $\mu$ is the dynamic viscosity.

Considering that the mass flow rate is small enough to activate the partial boundary velocity slip, the normal component of $\boldsymbol{U}$ on the wall is zero (see Fig.1b ), and the tangential component on the wall, denoted as $U_{w t}$, is a function of the stress vector tangent to the wall $\tau_{w t}$ :

$$
U_{w t}=\left\{\begin{array}{lr}
-S_{l} \tau_{w t}, & \text { linear } \\
-S_{n l} \tau_{w t}\left\|\tau_{w t}\right\|^{n-1}, & \text { nonlinear }
\end{array}\right.
$$

where $\left\|\tau_{w t}\right\|$ represents the $l^{2}$ norm of $\tau_{w t}$, the negative signs indicate $U_{w t}$ and $\tau_{w t}$ are in opposite directions, and $S_{l}$ and $S_{n l}$

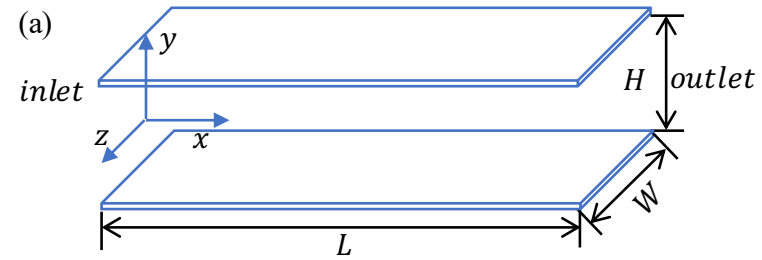

(b)

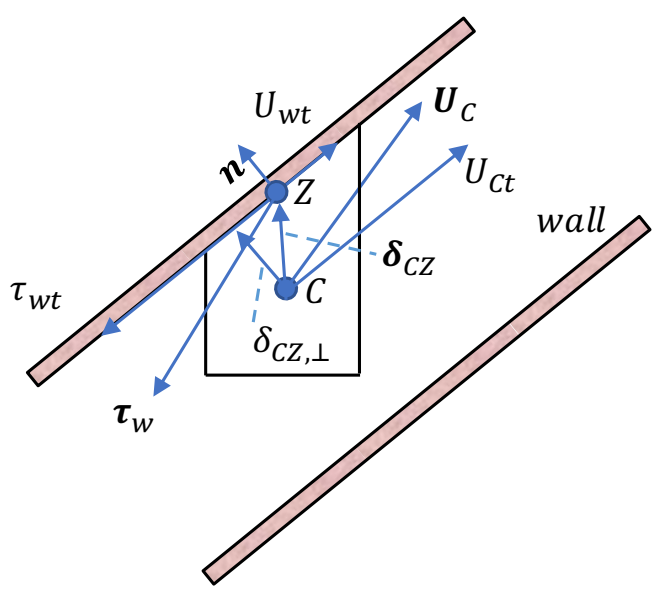

FIG. 1: Sketches of (a) the plane parallel channel and (b) the near wall velocity slip.

are the linear and nonlinear slip coefficients, respectively ${ }^{35,36}$. The present work will focus on the linear SBC, while the nonlinear counterpart commonly used for non-Newtonian fluids where the viscosity is dependent on shear rate ${ }^{37}$ can be implemented similarly.

For Newtonian flow, the fluid motion near the wall can be assumed as local Couette flow, therefore, the tangential wall stress vector $\tau_{w t}$ is

$\tau_{w t}=\mu \frac{\partial U_{t}}{\partial \delta_{C Z, \perp}}=\mu \frac{U_{w t}-U_{C t}}{\boldsymbol{\delta}_{C Z} \bullet \boldsymbol{n}}=\mu \frac{(\boldsymbol{I}-\boldsymbol{n} \otimes \boldsymbol{n}) \bullet\left(\boldsymbol{U}_{w}-\boldsymbol{U}_{C}\right)}{\boldsymbol{\delta}_{C Z} \bullet \boldsymbol{n}}$,

where $\mu$ is the dynamic viscosity, $C$ is the central point of the mesh cell next to the wall, $Z$ is the central point of a boundary face, $\delta_{C Z}$ is the vector between this two points and $\delta_{C Z, \perp}$ is the projection of $\boldsymbol{\delta}_{C Z}$ in the direction of $\boldsymbol{n}$. $\boldsymbol{U}_{w}$ and $\boldsymbol{U}_{C}$ are the velocity vector at $Z$ and $C$, respectively and $U_{w t}$ and $U_{C t}$ are their tangential components . $I$ is the third-order unit matrix and $\boldsymbol{n}$ is the normal vector to the boundary face.

Substituting Eq. (4) into Eq. (3a), the linear SBC can be obtained as

$$
U_{w t}=-L_{s l i p} \frac{(\boldsymbol{I}-\boldsymbol{n} \otimes \boldsymbol{n}) \bullet\left(\boldsymbol{U}_{w}-\boldsymbol{U}_{C}\right)}{\boldsymbol{\delta}_{C Z} \bullet \boldsymbol{n}},
$$

where $L_{\text {slip }}\left(L_{\text {slip }}=S_{l} \mu\right)$ is the velocity slip length ${ }^{38,39}$.

The tangential velocity of the fluid in the vicinity of the wall representing the Navier slip boundary conditions can be modelled via the following explicit scheme:

$$
U_{w t}^{i}=-L_{s l i p} \frac{(\boldsymbol{I}-\boldsymbol{n} \otimes \boldsymbol{n}) \bullet\left(\boldsymbol{U}_{w}^{i-1}-\boldsymbol{U}_{C}^{i-1}\right)}{\boldsymbol{\delta}_{C Z} \bullet \boldsymbol{n}},
$$


where the superscripts $i$ and $i-1$ denote values of corresponding quantities at the current or former time step, respectively. In the explicit scheme, relaxation is commonly implemented to improve the stability and convergence property when using large spatial or temporal intervals.

\section{B. Resistance coefficient derived from the exact solution of the NS equations}

The velocity profile for fully developed laminar flow through a plane parallel channel as shown in Fig. 1a with linear SBC can be obtained from Eq. (1) and Eq. (2):

$$
U_{x}=-\frac{1}{2 \mu} \frac{\mathrm{d} p}{\mathrm{~d} x}\left(\frac{H^{2}}{4}+L_{\text {slip }} H-y^{2}\right),
$$

where $H$ is the height of the channel, and this velocity profile is in agreement with references ${ }^{40}$. Then the average velocity can be calculated from

$$
U_{m}=\frac{\int_{\frac{-H}{2}}^{\frac{H}{2}} U_{x} \mathrm{~d} y}{H}=-\frac{1}{12 \mu} \frac{\mathrm{d} p}{\mathrm{~d} x}\left(H^{2}+6 L_{\text {slip }} H\right) .
$$

In general, the pressure loss over a streamwise length $L$ for all types (laminar or turbulent, circular or noncircular, smooth or rough surfaces, horizontal or inclined pipes) of fully developed internal flows can be represented as ${ }^{41}$

$$
\Delta p=K \frac{\rho U_{m}^{2}}{2},
$$

where $K$ is named the flow resistance coefficient, which measures the efficiency of the mass transfer.

Considering that the pressure gradient is constant, or $\Delta p / L=\mathrm{d} p / \mathrm{d} x$ and combining Eq. (8) and Eq. (9), $K$ for fully developed laminar flow in a plane parallel channel with linear SBC can be obtained as

$$
K=\frac{24}{\operatorname{Re}\left(1+6 \frac{L_{\text {slip }}}{H}\right)} \frac{L}{H},
$$

where $R e$ is the Reynolds number based on the average velocity and the channel height $H$.

Similarly the flow resistance coefficient in a fully developed laminar pipe flow with SBC can be obtained:

$$
K=\frac{64}{\operatorname{Re}\left(1+8 \frac{L_{\text {slip }}}{D}\right)} \frac{L}{D} .
$$

The first factor on the right of Eq. (10) and Eq. (11) represents the friction factor. Clearly in Eq. (11) when the no-slip condition is imposed $\left(L_{\text {slip }}=0\right)$, there is $K=64 / R e$, a well known formula for the macro-scale laminar pipe flow. Moreover, the wall friction coefficient $(f)$ can be defined as the ratio of the shear stress and fluid velocity at the wall, and subsequently there is $f=\mu / L_{\text {slip }}$ from Eq. (4) and Eq. (7). This indicates the wall friction coefficient is inversely proportional to the velocity slip length at constant fluid viscosity, in agreement with the experimental work $^{42}$.

\section{Resistance coefficient calculated from SLA}

As an alternative to the definition, it has been proposed that $K$ can be obtained from SLA for non-slip flow ${ }^{33}$ :

$$
K=\frac{T \dot{S}_{i r r, D}}{\rho \frac{U_{m}^{3}}{2} A}
$$

where $T$ is the temperature, $\dot{S}_{i r r, D}$ is the generation rate of entropy due to thermodynamic irreversibility, and $T \dot{S}_{i r r, D}$ corresponds to the rate of the exergy loss according to the Gouy-Stodola theorem ${ }^{43}$.

When the SLA method is used to calculate $K$ for the slip flow from the view of the balance of energy, the skin friction drag does negative work on the fluid (which is zero at the no-slip condition). In other words, the head loss of fluid is caused by two factors: viscous dissipation and skin friction drag. Therefore, the definition of the resistance coefficient should be adjusted to take into account this term as

$$
K=\frac{T \dot{S}_{i r r, D}+W_{d r a g}}{\rho \frac{U_{m}^{3}}{2} A},
$$

where $W_{\text {drag }}$ represents the contribution of the wall skin friction on fluid, and it can be calculated from Eq. (4) and Eq. (7) as

$$
W_{\text {drag }}=\left|\int_{S} \tau_{w t} \bullet U_{w t} \mathrm{~d} S\right|=\frac{72 \mu U_{m}^{2} L W H^{2} L_{\text {slip }}}{\left(H^{2}+6 L_{\text {slip }} H\right)^{2}} .
$$

Bejan $^{44}$ proposed that the volumetric rate of entropy generation consists of the volumetric rate of the heat transfer irreversibility and the fluid friction irreversibility. For steady, isothermal and incompressible channel flow without or with linear SBC considered in the present work, the local entropy generation rate is only from the fluid friction irreversibility and can be computed from

$$
\dot{S}_{i r r, D}^{\prime \prime \prime}=\frac{1}{T} \boldsymbol{\tau}: \nabla \boldsymbol{U}=144 \frac{\mu}{T} \frac{y^{2}}{\left(H^{2}+6 L_{s l i p} H\right)^{2}} U_{m}^{2} .
$$

The overall entropy production rate can be obtained by integrating the local entropy generation rate:

$$
\dot{S}_{i r r, D}=\int_{V} \dot{S}_{i r r, D}^{\prime \prime \prime} \mathrm{d} V=\frac{12 \mu W L}{T} \frac{H^{3} U_{m}^{2}}{\left(H^{2}+6 L_{\text {slip }} H\right)^{2}} .
$$

Substituting Eq. (14) and Eq. (16) and into Eq. (13), the flow resistance coefficient is

$$
K=\frac{24}{R e} \frac{1}{1+6 \frac{L_{\text {slip }}}{H}} \frac{L}{H},
$$

the same as Eq. (10) derived from the NS equations. Similarly the resistance coefficient in a fully developed laminar pipe flow with SBC can be obtained and it also matches that from the NS method (see Eq. (11)). 
TABLE I: Resistance coefficient for plane channel and circular pipe flow

\begin{tabular}{llll}
\hline \hline \multirow{2}{*}{ channel } & method & NS & SLA \\
& theoretical & 0.2427 & 0.2427 \\
pipe & simulational & 0.2403 & 0.2431 \\
& theoretical & 0.6009 & 0.6009 \\
\hline \hline
\end{tabular}

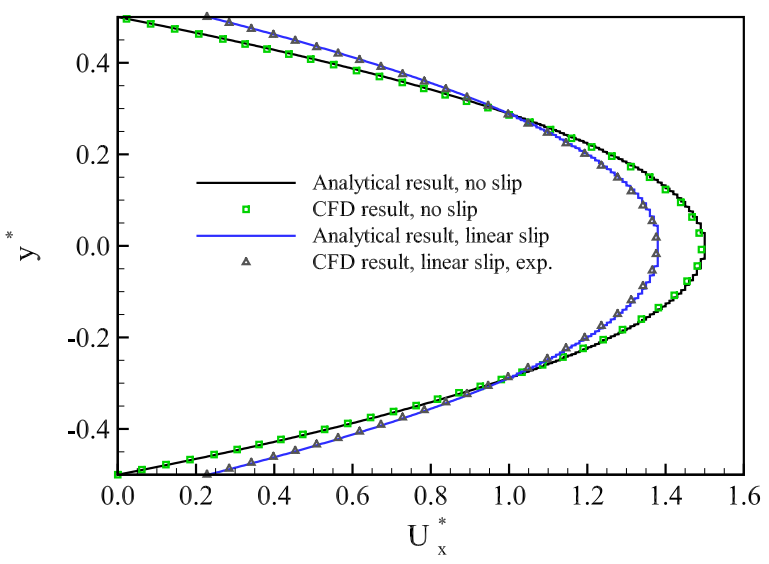

FIG. 2: Velocity profile in a channel flow, where $y^{*}=y / H$ and $U_{x}^{*}=U_{x} / U_{m}$.

\section{Validation}

Numerical simulations are performed to validate the derivations in Section IIC. The plane parallel channel shown in Fig. 1a with $L=30 \mathrm{~mm}, W=0.2 \mathrm{~mm}$ and $H=2 \mathrm{~mm}$ is adopted. A circular pipe with length and diameter $L=30 \mathrm{~mm}$ and $D=2 \mathrm{~mm}$, respectively, is also considered. The density of water is $\rho=997.05 \mathrm{~kg} / \mathrm{m}^{3}$, and its dynamic viscosity is $\mu=$ $8.90 \times 10^{-4} \mathrm{~Pa} \cdot \mathrm{s}$. The average velocity for no-slip or slip flow is $U_{m}=0.50929 \mathrm{~m} / \mathrm{s}$ and the slip length is $L_{\text {slip }}=10^{-4} \mathrm{~m}$. $\Delta t=10^{-4} s$ was chosen as the time step to ensure that the maximum Courant number is less than 1. The coupling of pressure and velocity is addressed by the PISO algorithm.

The streamwise velocity and coordinate system are nondimensionalised by average velocity $U_{m}$ and channel height $H$, respectively. Fig. 2 shows the dimensionless streamwise velocity profiles along the height direction of the plane channel (velocity distributions in a circular pipe are not shown here for conciseness). The simulation results are in excellent agreement with the analytical outcomes. Furthermore, the explicit scheme leads to well agreed velocity distributions. The resistance coefficients for fully developed slip flow in the plane parallel channel and the circular pipe calculated from the NS equations and the SLA approach are in good agreement with each other, as shown in Table I. This validates the analytical derivations presented in Section II C and demonstrate the accuracy of the aforementioned explicit scheme of the linear SBC.

\section{FLOW RESISTANCE IN A CHANNEL WITH AN SCC}

\section{A. Problem descriptions}

After investigating the efficiency of mass transfer in a straight channel, the flow resistance coefficient through an SCC as schematically illustrated in Fig. 3 will be examined using both the NS and the SLA approaches. The total flow resistance consists of the loss in the upstream large channel, downstream small channel and the SCC.

By definition, the flow resistance coefficient $K_{S C C}$ induced by the SCC can be written as

$$
K_{S C C}=\frac{1}{\rho \frac{U_{2}^{2}}{2}}\left[\Delta p-\Delta p_{u p, \circ}-\Delta p_{d o w n, \circ}+\frac{\rho\left(U_{1}^{2}-U_{2}^{2}\right)}{2}\right] \text {, }
$$

where $\Delta p_{u p, \circ}$ and $\Delta p_{\text {down }, \circ}$ denote the pressure loss of fully developed laminar flow in the upstream and downstream channel without SCC, respectively, and they can be determined from Eq. (9) and Eq. (10). $U_{2}$ is the average velocity at the outlet and will be used as the characteristic velocity, and $\Delta p$ is the pressure difference between inlet and outlet.

$K_{S C C}$ can also be obtained from the SLA method through re-writing Eq. (13)

$$
\begin{aligned}
K_{S C C} & =\frac{1}{\rho \frac{U_{2}^{3}}{2} A}\left(T \dot{S}_{\text {irr }, D, S C C}+W_{D, S C C}\right) \\
& =\frac{1}{\rho \frac{U_{2}^{3}}{2} A}\left[\left(T \dot{S}_{\text {irr }, D, \text { total }}+W_{D, \text { total }}\right)-\left(T \dot{S}_{\text {irr }, D, \text { up }, \circ}+\right.\right. \\
& \left.\left.W_{D, \text { up }, \circ}\right)-\left(T \dot{S}_{\text {irr }, D, \text { down }, \circ}+W_{D, \text { down }, \circ}\right)\right]
\end{aligned}
$$

where $T \dot{S}_{i r r, D, u p, \circ}$ and $T \dot{S}_{i r r, D, \text { down }, \circ}$ represent the entropy production rate of flow that is not affected by the SCC in the upstream and downstream channel, respectively. These terms are calculated from Eq. (16) when considering no-slip or linear SBC. $W_{D, u p, \circ}$ and $W_{D, \text { down,o }}$ are the work contributed by wall skin friction, and can be obtained from Eq. (14). $A$ is the area of the cross section of the downstream channel. Also, the overall flow resistance coefficient $K_{\text {total }}$ of this SCC could be determined directly from Eq. (13).

The height of the downstream channel $\mathrm{H}_{2}$ is set to $2 \times$ $10^{-5} \mathrm{~m}$ and used as the characteristic length, resulting in $L_{\text {slip }}^{*}=L_{\text {slip }} / H_{2} . H_{1} / H_{2}$ represents the contraction ratio and will be denoted as $R_{\text {area }}$. The upstream and downstream lengths of the channel are $L_{u p} / H_{2}=25$ and $L_{\text {down }} / H_{2}=$ 50 , respectively, which have been found to be long enough to accommodate the flow affected by the SCC. Moreover, the wall shear stress and the drag force work will be nondimensionalised by $\rho U_{2}^{2}$ and $\rho U_{2}^{3} H_{2}^{2}$, respectively. The time step for each case is adjusted to ensure that the maximum Courant number is less than 1 . 


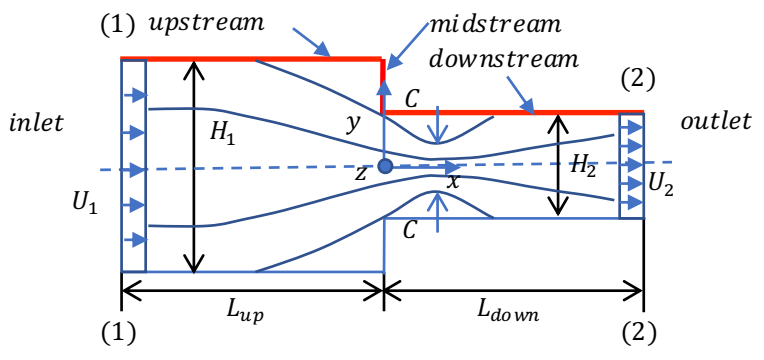

FIG. 3: Schematic of flow through an SCC.
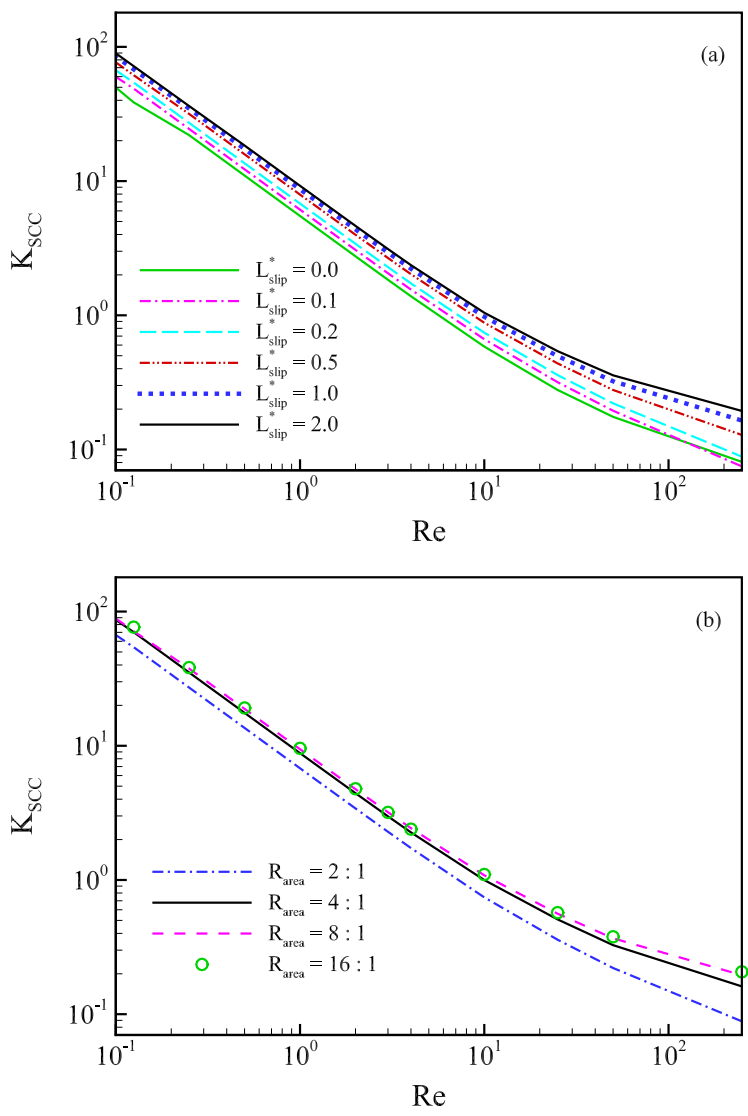

FIG. 4: Flow resistance coefficient of the SCC calculated from the SLA method at various $R e$ and $L_{\text {slip }}^{*}$. (a)

$$
R_{\text {area }}=2: 1 \text {, (b) } L_{\text {slip }}^{*}=0.2 \text {. }
$$

\section{B. The flow resistance coefficient}

The flow resistance coefficient $K_{S C C}$ of the SCC computed from Eq. (18) and Eq. (19) is shown in Fig. 4. Unlike the resistance coefficient in the macro turbulent flow which is only dependent on the cross section ratio and insensitive to $R e, K_{S C C}$ in the present micro channel drops rapidly with increasing $R e$. Fig. 4b shows the effect of the contraction ratio $R_{\text {area }}$ on the flow resistance coefficient $K_{S C C}$. At prescribed $R e$ and $L_{\text {slip }}^{*}$, one can find that $K_{S C C}$ increases slightly with the contraction ratio $R_{\text {area }}$. Through data fitting, the resistance coefficient over low $R e$ (e.g., $R e \leq 10)$ can be modelled as

$$
K_{S C C} \approx \frac{a L_{\text {slip }}^{* 3}+b L_{\text {slip }}^{* 2}+c L_{\text {slip }}^{*}+d}{\operatorname{Re}},
$$

where coefficients $(a, b, c, d)$ are constants at prescribed $R_{\text {area }}$, and are $(1.401,-5.518,7.299,5.488)$ and $(0.2283,-1.338,2.747,8.35)$ for $R_{\text {area }}=2: 1$ and $4: 1$, respectively. At $R_{\text {area }} \geq 8: 1,(a, b, c, d)$ converges to $(0.4454,-1.894,2.917,8.909)$, offering a formula for quick estimation of the resistance coefficient at small Reynolds numbers and large contraction ratios with a given velocity slip length. One should note that the fitting equation underestimates the resistance coefficient at larger $R e$.

In addition, $K_{S C C}$ can be transformed to the equivalent length of undisturbed fully developed downstream flow with the same flow resistance

$$
L_{\text {equi }}^{*}=\frac{L_{\text {equi }}}{H_{2}}=\frac{1}{24} \operatorname{Re} K_{S C C}\left(1+6 L_{\text {slip }}^{*}\right) .
$$

The dimensionless equivalent length $L_{\text {equi }}^{*}$ increases with respect to $L_{\text {slip }}^{*}$, but is insensitive to $R e$ when $R e$ is small enough as shown in Fig. 5, where $R_{\text {area }}$ is fixed at 4:1 and the numerically computed $K_{S C C}$ is adopted. This can be also demonstrated analytically by substituting Eq. (20) into Eq. (21), which results in a $R e$-independent $L_{\text {equi }}^{*}$. However at higher $\operatorname{Re}$ (e.g. $R e \geq 10$ ), the analytical fitting equation underestimates the resistance coefficient $K_{S C C}$ and consequently $L_{\text {equi }}^{*}$, as confirmed by the numerical results in Fig. 5. Variations of $L_{\text {equi }}^{*}$ at other $R_{\text {area }}$ are similar and are not shown here.

Above we reported effects of $R e, L_{\text {slip }}^{*}$ and $R_{\text {area }}$ on the flow resistance coefficient $K_{S C C}$ and dimensionless equivalent length $L_{\text {equi }}^{*}$. In the following sections, the fluid physics will be explored and efforts will be focused on the two sources of the flow resistance, i.e., drag force work and viscous dissipation loss.

\section{Wall shear stress vector and drag force work}

The dimensionless drag force work of the SCC, $W_{D, S C C}^{*}$, increases with $R e$ number for velocity slip flow as shown in Fig. 6. Furthermore, there is a critical dimensionless velocity slip length $\left(L_{\text {slip }, c r i}^{*} \approx 0.4\right.$ for all the four $R_{\text {area }}$ studied) where $W_{D, S C C}^{*}$ reaches its minimum. One should note that $W_{D, S C C}^{*}$ is almost always less than 0 (the reason will be illustrated later), indicating that the drag force work tends to reduce the flow resistance coefficient of the SCC according to the definition in Eq. (19).

Fig. 7 describes how $L_{\text {slip }}^{*}$ affects the dimensionless wall velocity and shear stress distributions for the upstream, midstream and downstream of the SCC shown in Fig. 3 at $R_{\text {area }}=$ $4: 1$ (results for other contraction ratios are similar and are not shown here for brevity). Away from the SCC, the dimensionless velocity and wall shear stress maintain constant values that match the velocity and shear stress in the straight channel flow from analytical calculations. The magnitude of near wall velocity enlarges with increasing $L_{\text {slip }}^{*}$. Conversely, the 


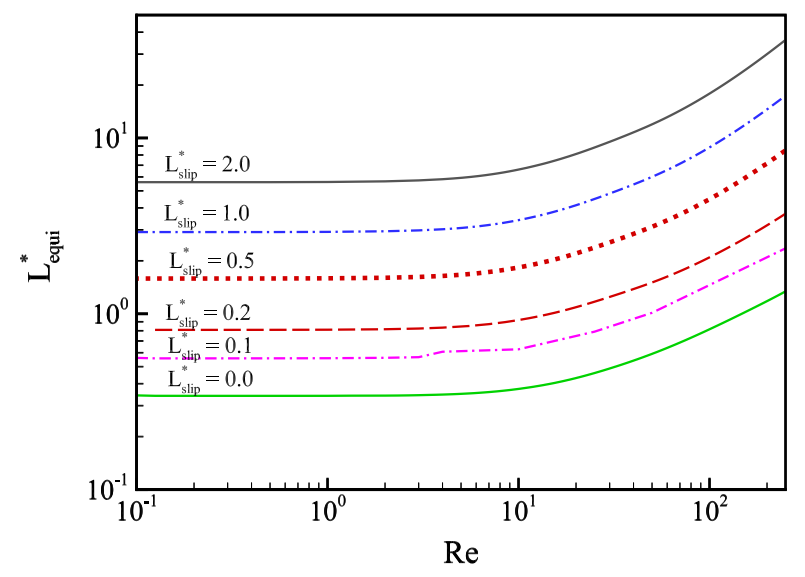

FIG. 5: The equivalent length of the resistance coefficients at various $R e$ and $L_{\text {slip }}^{*}$ at $R_{\text {area }}=4: 1$.

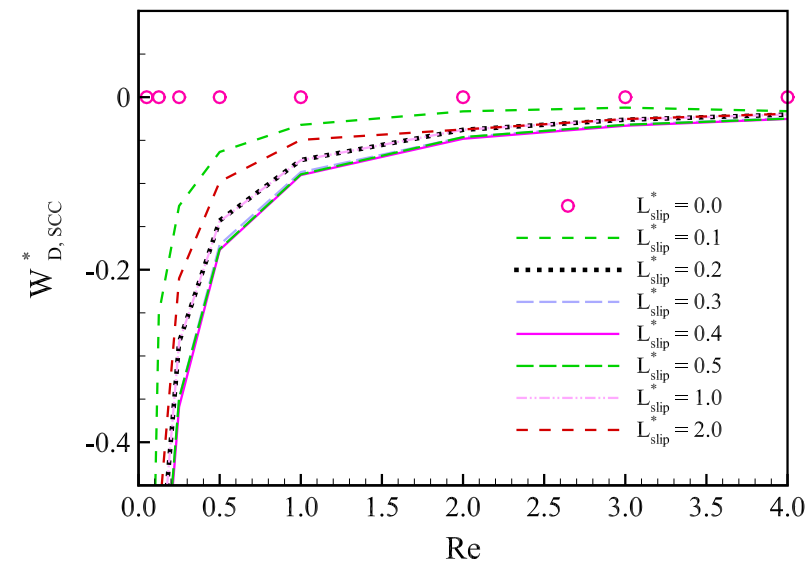

FIG. 6: Wall shear tangential stress work of the SCC at various $R e$ and $L_{\text {slip }}^{*}$ at $R_{\text {area }}=4: 1$.

amplitude of the upstream and downstream wall shear stress $\tau_{x}^{*}$ for slip flow are far less than $\left|\tau_{x}^{*}\right|$ for no-slip flow at a prescribed $R e$, and this is in agreement with the experimental observation $^{42}$.

The dimensionless drag force work per unit area $w_{d}^{*}$ for the SCC with $R_{\text {area }}=4: 1$ is plotted in Fig. 8 to explain the negative value of $W_{D, S C C}^{*}$ mentioned above. In terms of the dimensionless tangential wall shear stress work of the SCC in Fig. 6, $W_{D, S C C}^{*}$ can be rewritten as

$W_{D, S C C}^{*}=\left(W_{D, u p}^{*}-W_{D, u p, \circ}^{*}\right)+W_{D, \text { mid }}^{*}+\left(W_{D, \text { down }}^{*}-W_{D, \text { down }, \circ}^{*}\right)$

The region of the downstream channel affected by the SCC is very small according to Fig. 8 and $w_{d, \text { down }}^{*}$ is almost constant rendering the third term of the right side of Eq. (22) close to 0 . Furthermore, $W_{D \text {,mid }}^{*}$ is essentially very small resulting in $W_{D, S C C}^{*} \approx W_{D, u p}^{*}-W_{D, u p, \circ}^{*}$. From Fig. 8 , one can observe that $w_{d, u p}^{*}$ affected by the SCC is equal to $w_{d, u p, \circ}^{*}$ at most part of the upstream channel, and becomes smaller than $w_{d, u p, \circ}^{*}$ at the end of the upstream channel. This leads to a negative value of $W_{D, S C C}^{*}$, as observed before in Fig. 6 .
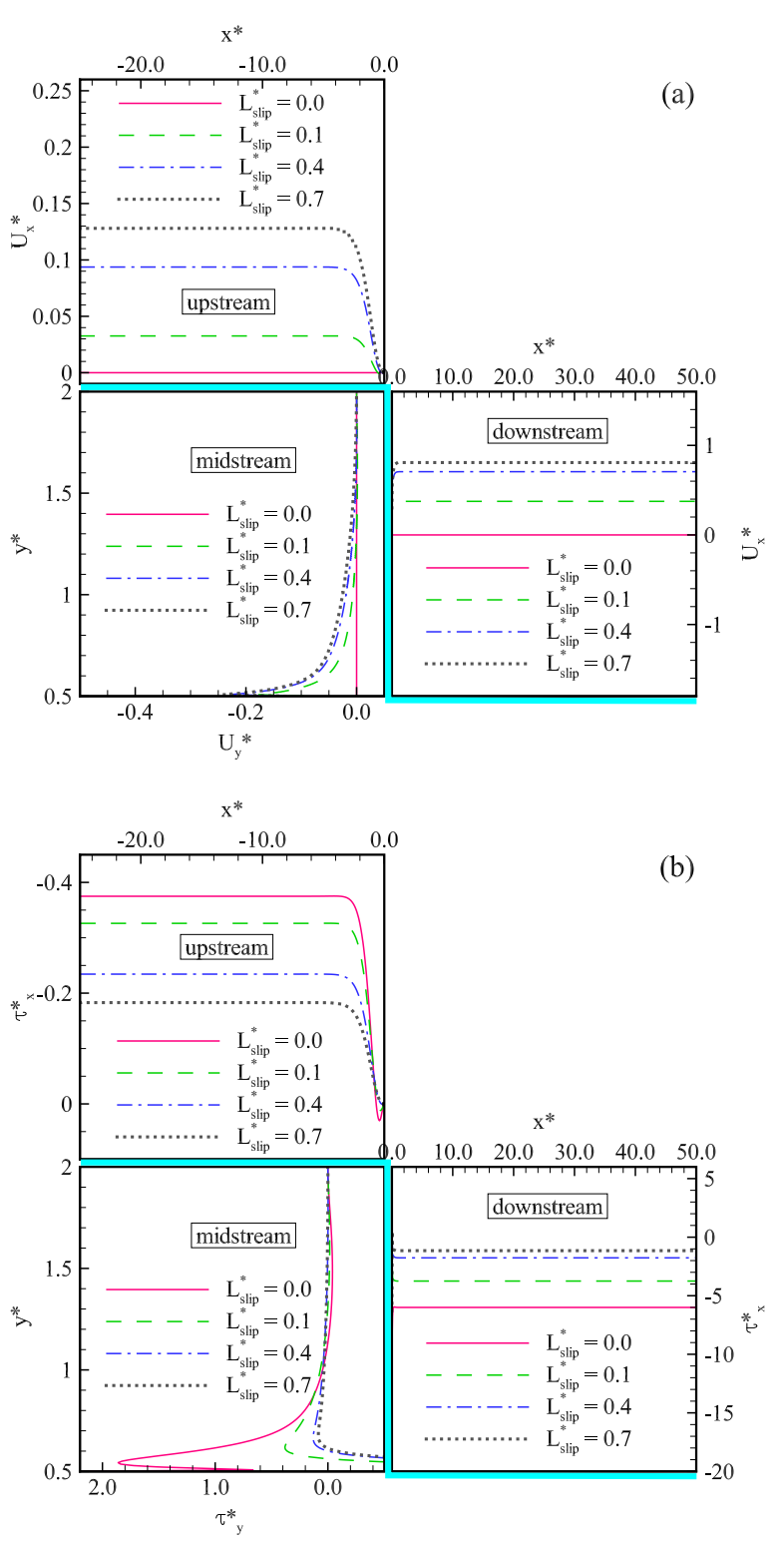

FIG. 7: Streamwise (upstream and downstream sections) and vertical (midstream section) velocity on the wall (a) and shear stress (b) profiles at various $L_{\text {slip }}^{*}$ at $R_{\text {area }}=4: 1, R e=1$ (the shallow green edges represent upstream, midstream and downstream channel as illustrated in Fig. 3).

\section{Disturbed region and local entropy generation rate}

To examine the range of regions affected by the SCC, a nondimensional surface integral of the local entropy production rate is defined as

$$
\dot{S}_{i r r, D}^{\prime * *}=\frac{\dot{S}_{i r r, D}^{\prime}}{\dot{S}_{i r r, D, \circ}^{\prime}}=\frac{\int_{A} \dot{S}_{i r r, D}^{\prime \prime \prime} \mathrm{d} A}{\int_{A} \dot{S}_{i r r, D, \circ}^{\prime \prime \prime} \mathrm{d} A},
$$

where $A$ is an arbitrary cross section area. $\dot{S}_{i r r, D, \circ}^{\prime \prime \prime}$ represents the local entropy production rate of flow without SCC and its surface integral can be formulated based on Eq. (16). 


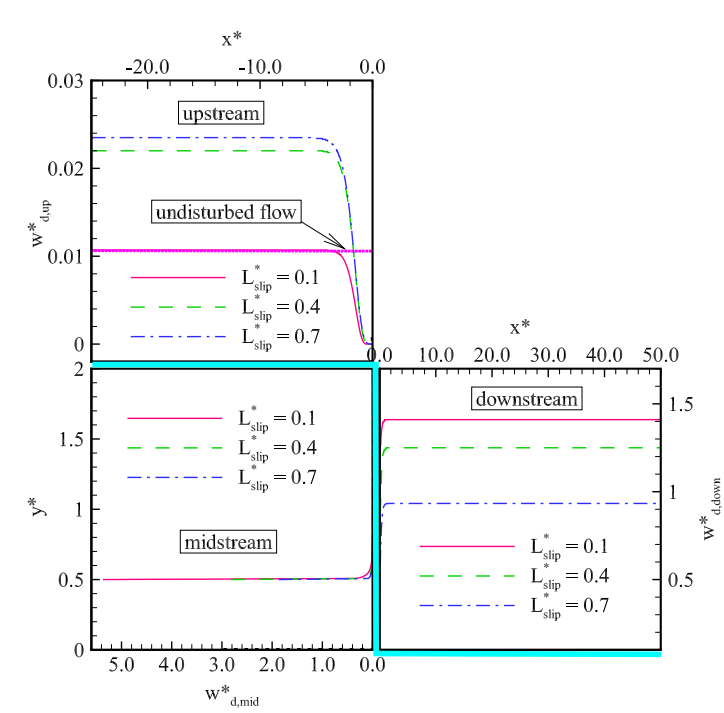

FIG. 8: Drag work per unit area at various $L_{\text {slip }}^{*}$ when $R_{\text {area }}=4: 1$ and $R e=1$ (the shallow green edges represent upstream, midstream and downstream channel as illustrated in Fig. 3).

The non-dimensional length of upstream and downstream channel domain affected by the SCC can be defined as

$$
L_{u p, d i s t}^{*}=\frac{\left|0-x_{\text {up,dist }}\right|}{H_{2}}, L_{\text {down,dist }}^{*}=\frac{\left|x_{\text {down, dist }}-0\right|}{H_{2}},
$$

where $x_{u p, d i s t}$ and $x_{\text {down,dist }}$ are $x$ coordinates of the disturbed points where $\left.\dot{S}_{i r, D}^{\prime \prime *}\right|_{x=x_{u p, d i s t}}=0.95$ and $\left.\dot{S}_{i r r, D}^{\prime \prime *}\right|_{x=x_{\text {down,dist }}}=$ 0.95 , respectively ${ }^{33} .0$ represents the $x$ coordinate of the position of the SCC, where the area of the cross section changes abruptly. As shown in Fig. 9, $L_{u p, d i s t}^{*}$ and $L_{\text {down,dist }}^{*}$ are almost independent on $R e$ and increase with $L_{\text {slip }}^{*}$. In addition, $L_{\text {up,dist }}^{*}$ rises conspicuously and $L_{\text {down,dist }}^{*}$ remains almost constant with increasing $R_{\text {area }}$.

Contours of $T \dot{S}_{i r r, D}^{\prime \prime \prime}$ are shown in Fig. 10 from which we can observe the viscous dissipation loss of fluid at every point in the flow field and the effects of $R e, L_{\text {slip }}^{*}$ and $R_{\text {area }}$. Comparing Fig. 10a with Fig. 10b, the viscous dissipation loss further concentrates on the corner as marked by the green circles in these two subfigures at larger $R e$. One interesting observation is that an "ear" type dissipation loss region appears at the beginning section of the downstream channel for slip flow, as marked by the green circle in Fig. 10c.

Moreover, the local entropy production rate of fluid in the SCC consists of six sections, mainly including upstream and downstream channels and $90^{\circ}$ corners, etc (see Fig. 10d). Conspicuously, $T \dot{S}_{i r r, D}^{\prime \prime \prime}$ for flow of the upstream and downstream channel affected by the SCC increases from the center line of the SCC and peaks at the wall (see the arrow (1) and (6) in Fig. 10d), suggesting that the viscous dissipation loss minimizes at the center line and enlarges along the height direction of upstream and downstream channels. $T \dot{S}_{i r r, D}^{\prime \prime \prime}$ for the $90^{\circ}$ corner (4) increases along the direction departing from the corner,
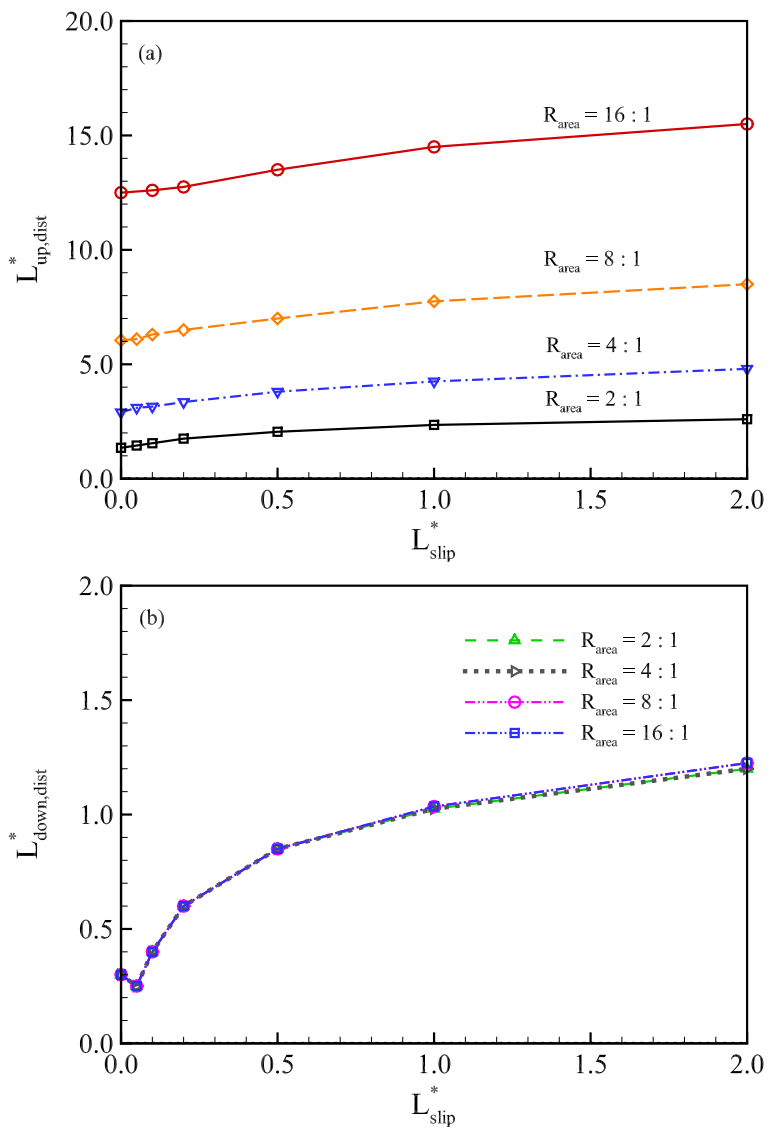

FIG. 9: Dimensionless length of the disturbed domain at $R e=0.5$. (a) upstream, (b) downstream.

and the variation trend is opposite to the other $90^{\circ}$ corner (5) where the local entropy generation rate or flow resistance is the maximum in the whole flow field. The growth gradient for $T \dot{S}_{i r r, D}^{\prime \prime \prime}$ at these two corners rises dramatically at larger $R e$. An interesting point is that there is a "source" (surrounded by the green circle in Fig. 10d) of $T \dot{S}_{i r r, D}^{\prime \prime \prime}$, from which the flow resistance increases along with opposite directions (2) and (3) in Fig. 10d).

\section{CONCLUSIONS}

This work addresses the flow resistance coefficient in flow passing through a pipe or channel with or without a sudden contraction component at low Reynolds number conditions with a slip wall velocity boundary condition which is implemented using the explicit scheme.

The resistance coefficients of the fully developed channel and pipe flow at low $R e$ with linear Navier velocity slip boundary condition are solved with the NS equations and the second law analysis (SLA) approach which is extended to determine and visualize the flow resistance loss of velocity slip flow after considering the work contributed by wall drag force.

Then, numerical simulations are also performed for these two geometry models and the obtained resistance coefficients 

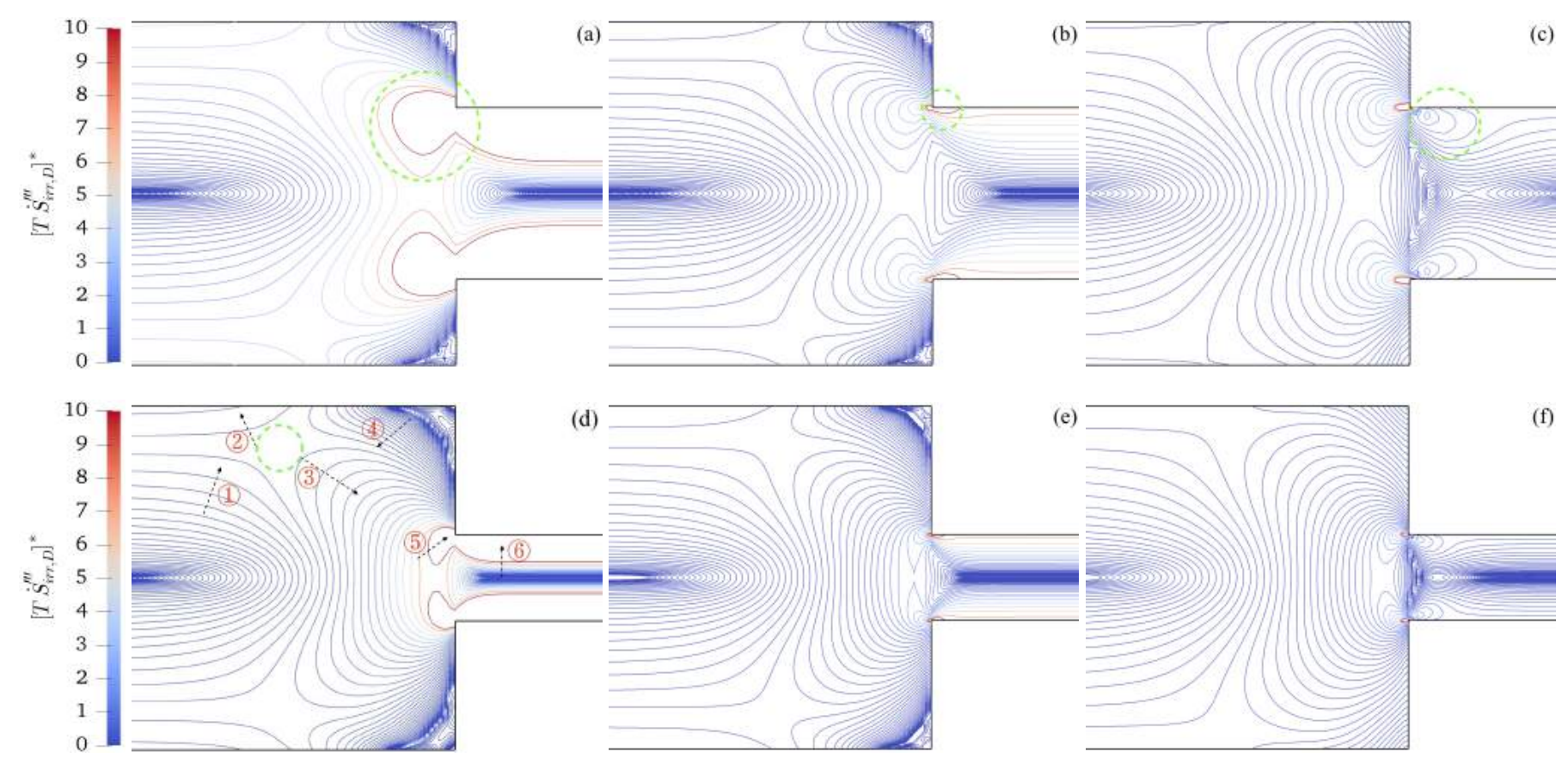

(f)
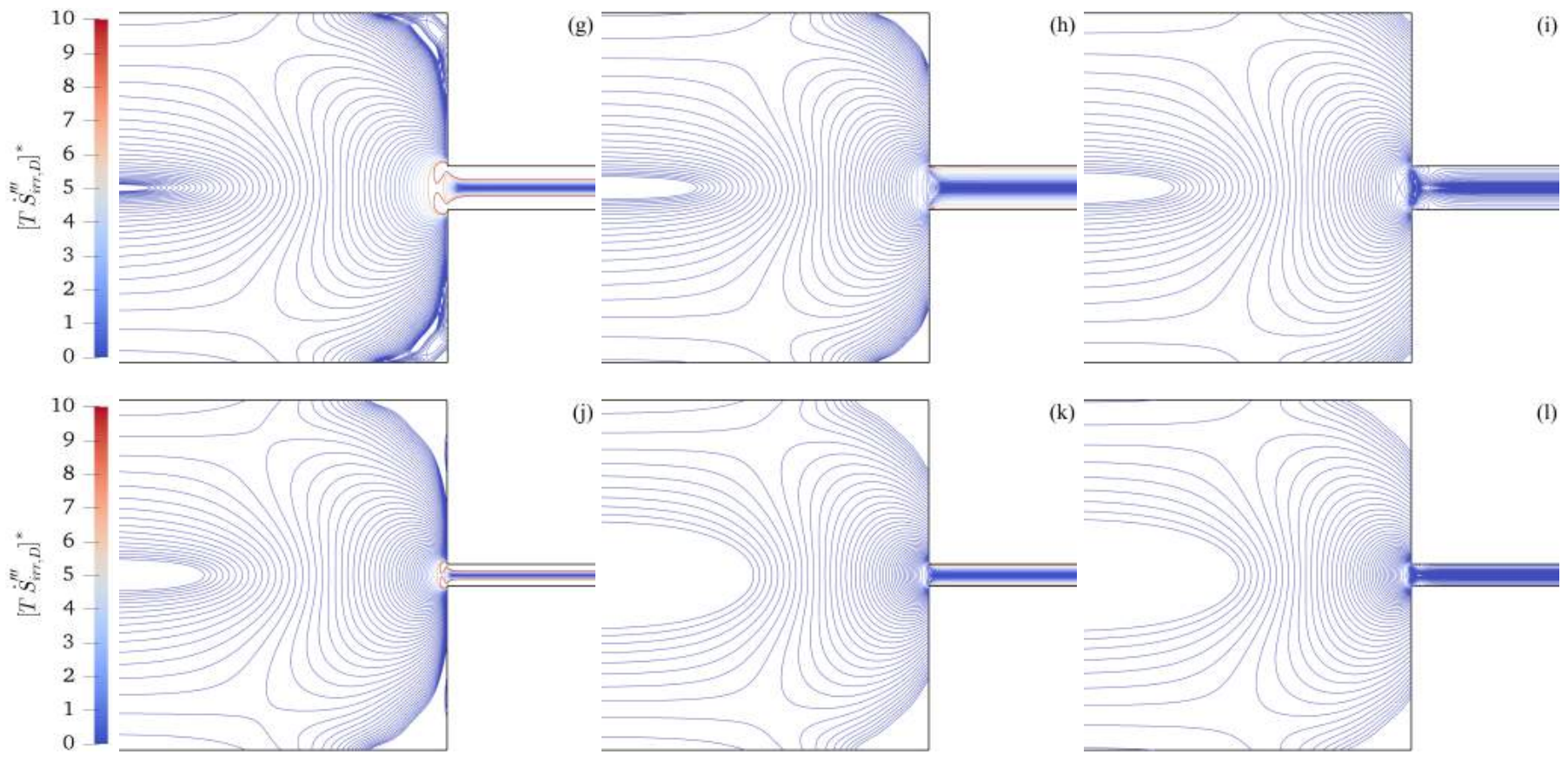

(k)

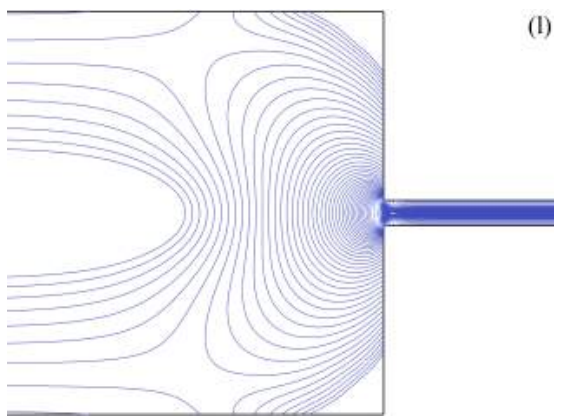

FIG. 10: Contours of local entropy generation rate $\dot{S}_{i r r, D}^{\prime \prime \prime}$ at per unit temperature non-dimensionalised by $\rho U_{2}^{3} / H_{2}$ at various $R e$, $L_{\text {slip }}$ and $R_{\text {area }}$. (a) $R e=0.5, L_{\text {slip }}^{*}=0.0, R_{\text {area }}=2: 1$, (b) $\operatorname{Re}=4.0, L_{\text {slip }}^{*}=0.0, R_{\text {area }}=2: 1$, (c) $\operatorname{Re}=4.0, L_{\text {slip }}^{*}=0.5$, $R_{\text {area }}=2: 1$, (d) $R e=0.5, L_{\text {slip }}^{*}=0.0, R_{\text {area }}=4: 1$, (e) $R e=4.0, L_{\text {slip }}^{*}=0.0, R_{\text {area }}=4: 1$, (f) $R e=4.0, L_{\text {slip }}^{*}=0.5$, $R_{\text {area }}=4: 1,(\mathrm{~g}) \operatorname{Re}=0.5, L_{\text {slip }}^{*}=0.0, R_{\text {area }}=8: 1$, (h) $R e=4.0, L_{\text {slip }}^{*}=0.0, R_{\text {area }}=8: 1$, (i) $\operatorname{Re}=4.0, L_{\text {slip }}^{*}=0.5$, $R_{\text {area }}=8: 1,(\mathrm{j}) \operatorname{Re}=0.5, L_{\text {slip }}^{*}=0.0, R_{\text {area }}=16: 1,(\mathrm{k}) \operatorname{Re}=4.0, L_{\text {slip }}^{*}=0.0, R_{\text {area }}=16: 1,(1) \operatorname{Re}=4.0, L_{\text {slip }}^{*}=0.5$, $R_{\text {area }}=16: 1$. 
are in good agreement with analytical outcomes.

Effects of $R e$ and dimensionless velocity slip length $\left(L_{\text {slip }}^{*}\right)$ on the flow resistance coefficient of laminar flow through the SCC with various contraction ratios $\left(R_{\text {area }}\right)$ are revealed based on the aforementioned NS equations and SLA method after conducting a series of numerical simulations.

At $R e \leq 10$, the flow resistance coefficient for the SCC, denoted as $K_{S C C}$, increases monotonously with $L_{\text {slip }}^{*}$ and $1 / R e$ and nonlinearly with $R_{\text {area }}$, and at $R_{\text {area }} \geq 8$, the resistance coefficient can be modelled as $K_{S C C}=\left(0.4454 L_{\text {slip }}^{* 3}-\right.$ $\left.1.894 L_{\text {slip }}^{* 2}+2.917 L_{\text {slip }}^{*}+8.909\right) /$ Re.

Physically, the flow resistance of fluids results from two factors: wall drag force work (equal to 0 for no-slip flow) and viscous dissipation in the flow field related to the local entropy generation rate. These two terms are computed and analyzed individually to illustrate the fluid physics underpinning the flow resistance.

The amplitude of the negative drag work $\left(W_{D, S C C}^{*}\right)$ decreases monotonously at larger $R e$. Also, there is a critical velocity slip length at which the amplitude of $W_{D, S C C}^{*}$ reaches maximum. In terms of fluid internal viscous dissipation, it minimizes at the center line and peaks at the wall of the upstream and the downstream channel. Moreover, the dissipation loss concentrates around the end section of the upstream channel of the SCC.

Both the dimensionless lengths of the disturbed region of the upstream and downstream channels are almost independent on $R e$ and enlarge at larger $L_{\text {slip }}^{*}$. With increasing $R_{\text {area }}$, the former rises remarkably while the latter remains nearly a constant value.

\section{ACKNOWLEDGMENTS}

We gratefully acknowledge the financial support from China Scholarship Council (CSC). This project has received funding from the European Union's Horizon 2020 research and innovation programme under the Marie SkłodowskaCurie grant agreement No 777717.

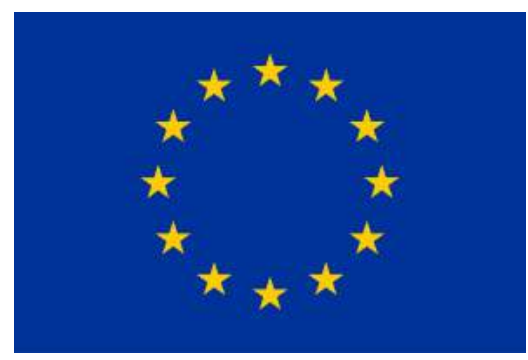

\section{DATA AVAILABILITY}

The data that support the findings of this study are available from the corresponding author upon reasonable request.

\section{REFERENCES}

${ }^{1}$ W. Y. Lee, M. Wong, and Y. Zohar, "Microchannels in series connected via a contraction/expansion section," Journal of Fluid Mechanics 459, 187-206 (2002).

${ }^{2}$ M. Sheikholeslami, R.-u. Haq, A. Shafee, Z. Li, Y. G. Elaraki, and I. Tlili, "Heat transfer simulation of heat storage unit with nanoparticles and fins through a heat exchanger," International Journal of Heat and Mass Transfer 135, 470-478 (2019).

${ }^{3}$ A. Radwan, S. Ookawara, S. Mori, and M. Ahmed, "Uniform cooling for concentrator photovoltaic cells and electronic chips by forced convective boiling in 3d-printed monolithic double-layer microchannel heat sink," Energy conversion and management 166, 356-371 (2018).

${ }^{4} \mathrm{H}$. Darcy, Recherches expérimentales relatives au mouvement de l'eau dans les tuyaux, Vol. 1 (Impr. Impériale, 1857).

${ }^{5} \mathrm{D}$. Valougeorgis, "The friction factor of a rarefied gas flow in a circular tube," Physics of Fluids 19, 091702 (2007).

${ }^{6} \mathrm{~J}$. Tao, "Critical instability and friction scaling of fluid flows through pipes with rough inner surfaces," Physical review letters 103, 264502 (2009).

${ }^{7}$ E. Calzetta, "Friction factor for turbulent flow in rough pipes from heisenberg's closure hypothesis," Physical Review E 79, 056311 (2009).

${ }^{8}$ R. T. Cerbus, C.-c. Liu, G. Gioia, and P. Chakraborty, "Laws of resistance in transitional pipe flows," Physical review letters 120, 054502 (2018).

${ }^{9}$ L. F. Moody, "Friction factors for pipe flow," Trans. Asme 66, 671-684 (1944).

${ }^{10} \mathrm{~B}$. Kim, "An experimental study on fully developed laminar flow and heat transfer in rectangular microchannels," International Journal of Heat and Fluid Flow 62, 224-232 (2016).

${ }^{11}$ P. J. Oliveira, F. Pinho, and A. Schulte, "A general correlation for the local loss coefficient in newtonian axisymmetric sudden expansions," International journal of heat and fluid flow 19, 655-660 (1998).

${ }^{12}$ W. Qu, G. M. Mala, and D. Li, "Pressure-driven water flows in trapezoidal silicon microchannels," International Journal of Heat and Mass Transfer 43, 353-364 (2000).

${ }^{13}$ J. Ou, B. Perot, and J. P. Rothstein, "Laminar drag reduction in microchannels using ultrahydrophobic surfaces," Physics of fluids 16, 4635-4643 (2004).

${ }^{14}$ C.-H. Choi, U. Ulmanella, J. Kim, C.-M. Ho, and C.-J. Kim, "Effective slip and friction reduction in nanograted superhydrophobic microchannels," Physics of fluids 18, 087105 (2006).

${ }^{15}$ P. Joseph, C. Cottin-Bizonne, J.-M. Benoit, C. Ybert, C. Journet, P. Tabeling, and L. Bocquet, "Slippage of water past superhydrophobic carbon nanotube forests in microchannels," Physical review letters 97, 156104 (2006).

${ }^{16}$ T. Ewart, P. Perrier, I. A. Graur, and J. G. Méolans, "Mass flow rate measurements in a microchannel, from hydrodynamic to near free molecular regimes," Journal of fluid mechanics 584, 337-356 (2007).

${ }^{17}$ A. Demsis, B. Verma, S. Prabhu, and A. Agrawal, "Experimental determination of heat transfer coefficient in the slip regime and its anomalously low value," Physical Review E 80, 016311 (2009).

${ }^{18}$ C.-M. Ho and Y.-C. Tai, "Micro-electro-mechanical-systems (mems) and fluid flows," Annual review of fluid mechanics 30, 579-612 (1998).

${ }^{19}$ C. Clasen and G. H. McKinley, "Gap-dependent microrheometry of complex liquids," Journal of Non-Newtonian Fluid Mechanics 124, 1-10 (2004).

${ }^{20} \mathrm{P}$. A. Thompson and S. M. Troian, "A general boundary condition for liquid flow at solid surfaces," Nature 389, 360 (1997).

${ }^{21}$ J. Péméja, B. Géraud, C. Barentin, and M. Le Merrer, "Wall slip regimes in jammed suspensions of soft microgels," Physical Review Fluids 4, 033301 (2019).

${ }^{22}$ J. Seo and A. Mani, "On the scaling of the slip velocity in turbulent flows over superhydrophobic surfaces," Physics of Fluids 28, 025110 (2016).

${ }^{23}$ G. Chattopadhyay, R. Usha, and K. C. Sahu, "Core-annular miscible twofluid flow in a slippery pipe: A stability analysis," Physics of Fluids 29, 097106 (2017).

${ }^{24} \mathrm{~K}$. Watanabe, Y. Udagawa, and H. Udagawa, "Drag reduction of newtonian fluid in a circular pipe with a highly water-repellent wall," Journal of Fluid Mechanics 381, 225-238 (1999).

${ }^{25}$ D. Gloss and H. Herwig, "Microchannel roughness effects: a close-up view," Heat Transfer Engineering 30, 62-69 (2009). 
${ }^{26}$ R. G. Owens and T. N. Phillips, Computational rheology, Vol. 14 (World Scientific, 2002).

${ }^{27}$ A. Sunarso, T. Yamamoto, and N. Mori, "Numerical analysis of wall slip effects on flow of newtonian and non-newtonian fluids in macro and micro contraction channels," Journal of fluids engineering 129, 23-30 (2007).

${ }^{28} \mathrm{D}$. V. Boger and K. Walters, Rheological phenomena in focus, Vol. 4 (Elsevier, 2012).

${ }^{29}$ G. Celata, G. Morini, V. Marconi, S. McPhail, and G. Zummo, "Using viscous heating to determine the friction factor in microchannels-an experimental validation," Experimental thermal and fluid science 30, 725-731 (2006).

${ }^{30}$ G. F. Naterer and J. A. Camberos, Entropy Based Design and Analysis of Fluids Engineering Systems (CRC press, 2008).

${ }^{31}$ A. Bejan and J. Kestin, "Entropy generation through heat and fluid flow," Journal of Applied Mechanics 50, 475 (1983).

${ }^{32} \mathrm{~S}$. Bo and A. Celani, "Entropic anomaly and maximal efficiency of microscopic heat engines," Physical Review E 87, 050102 (2013).

${ }^{33}$ H. Herwig and B. Schmandt, "How to determine losses in a flow field: A paradigm shift towards the second law analysis," Entropy 16, 2959-2989 (2014).

${ }^{34}$ S. Alokaily, K. Feigl, and F. X. Tanner, "Characterization of peristaltic flow during the mixing process in a model human stomach," Physics of Fluids 31, 103105 (2019).

${ }^{35}$ C. Navier, "Memoiresur les lois du movement des fluids, mem. acad. r," Sci. Inst. Fr 6, 389-416 (1823).
${ }^{36}$ C. Fernandes, L. L. Ferrás, F. Habla, O. S. Carneiro, and J. M. Nóbrega, "Implementation of partial slip boundary conditions in an open-source finite-volume-based computational library," Journal of Polymer Engineering 39, 377-387 (2019).

${ }^{37}$ K. Feigl, A. Baniabedalruhman, F. X. Tanner, and E. J. Windhab, "Numerical simulations of the breakup of emulsion droplets inside a spraying nozzle," Physics of Fluids 28, 123103 (2016).

${ }^{38}$ C.-O. Ng and R. Sun, "Pressure loss in channel flow resulting from a sudden change in boundary condition from no-slip to partial-slip," Physics of Fluids 29, 103603 (2017).

${ }^{39}$ C.-O. Ng and W. Xie, "End loss for stokes flow through a slippery circular pore in a barrier of finite thickness," Physics of Fluids 30, 103604 (2018).

${ }^{40}$ Q. S. Kwing-So Choi, Xuerui Mao, "An analytical solution of convective heat transfer in microchannel or nanochannel," International Communications in Heat and Mass Transfer (under review).

${ }^{41} \mathrm{M}$. Sheikholeslami and D. Ganji, "Heat transfer enhancement in an air to water heat exchanger with discontinuous helical turbulators; experimental and numerical studies," Energy 116, 341-352 (2016).

${ }^{42}$ Y. Wu, M. Cai, Z. Li, X. Song, H. Wang, X. Pei, and F. Zhou, "Slip flow of diverse liquids on robust superomniphobic surfaces," Journal of colloid and interface science 414, 9-13 (2014).

${ }^{43}$ H. D. Baehr and S. Kabelac, "Thermodynamik," Thermodynamik: Grundlagen und technische Anwendungen, Springer-Lehrbuch. ISBN 978-3-64200555-8. Springer-Verlag Berlin Heidelberg, 2009 (2009).

${ }^{44}$ A. Bejan, Entropy generation through heat and fluid flow (Wiley, 1982). 